\title{
PRODUCCIÓN DE ARROZ EN CHIRIQUÍ EN RELACIÓN CON LA SUPERFICIE SEMBRADA Y SU APORTE AL CONSUMO NACIONAL
}

\author{
Rice production in Chiriqui in relation to the seeded surface and its \\ contribution to national consumption
}

\author{
René Caballero Santamaría \\ Profesor regular, Facultad de Administración de Empresas y Contabilidad, Universidad de Panamá \\ rcaballero@gmail.com
}

\begin{abstract}
RESUMEN
Se analiza la producción de arroz en la Provincia de Chiriquí medido en quintales en cáscara. Se toma como referencia el período 1990 - 2017. Como fuente primaria para este análisis se extrajeron los datos generados por la Contraloría General de la República de Panamá, sección del Instituto Nacional de Estadística y Censo (INEC), fuente de internet https://www.contraloria.gob.pa/inec/. Tomando como marco de referencia el supuesto que "La cantidad de arroz cosechada en la Provincia de Chiriquí depende de la superficie sembrada y representa un aporte significativo al consumo nacional". Para sustentar esta conjetura (hipótesis) con los datos obtenidos se procedió a calcular las medidas de tendencia central, dispersión, forma y correlación. Los valores de la serie de tiempo han sido analizados a la luz de los conceptos y procedimientos estadísticos, que reflejan la existencia de una relación directamente proporcional entre la superficie sembrada y la cosecha de arroz generada. Además, se elaboró un modelo de regresión simple, que ha permitido constatar la hipótesis con el estadístico de correlación de Pearson ( $r=\mathbf{0 . 9 2 4}$ ) que evidencia la asociación de las variables y de Durbin Watson (D.W. = 1.918) que da como resultado que el modelo es robusto para predecir. También, se determinó que la Provincia de Chiriquí aporta $19.17 \%$ de su producción al consumo nacional.
\end{abstract}

Palabras claves: Arroz, superficie, modelo, regresión, proyección.

\begin{abstract}
Rice production in the Province of Chiriquí measured in quintals in the shell is analyzed. The period 1990-2017 is taken as a reference. As a primary source for this analysis, the data generated by the Comptroller General of the Republic of Panama, section of the National Institute of Statistics and Census (INEC), internet source https: // www.contraloria.gob.pa/inec/. Taking as a frame of reference the assumption that "The amount of rice harvested in the Province of Chiriquí depends on the area planted and represents a significant contribution to national consumption." To support this conjecture (hypothesis) with the data obtained, the measures of central tendency, dispersion, form and correlation were calculated. The time series values have been analyzed in the light of statistical concepts and procedures, which reflect the existence of a directly proportional relationship between the area planted and the rice crop generated. In addition, a simple regression model was developed, which has allowed us to verify the hypothesis with the Pearson correlation statistic $(\mathbf{r}=\mathbf{0 . 9 2 4 )}$ that evidences the association of the variables and Durbin Watson $(D W=1.918)$ that results in the Model is robust to predict. Also, it was determined that the Province of Chiriquí contributes $19.17 \%$ of its production to national consumption.
\end{abstract}

Keywords: Rice, surface, model, regression, projection. 


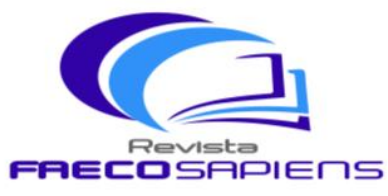

Reviste

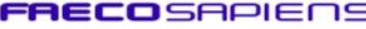

ताT Volumen 3 - Número 1 Enero-Junio 2020

Recibido: 18/10/19 Aceptado: 25/11/19 pp. 21-40 Indexada

\section{INTRODUCCIÓN}

La investigación contempla un estudio sobre la relación que existe entre las hectáreas sembradas de arroz y la producción generada, con esto se busca conformar un modelo que permita a los agricultores de la Provincia de Chiriquí y al estado, determinar anticipadamente la producción esperada. Con esta información el estado puede adelantar los posibles requerimientos para cubrir la demanda del país, por ser este producto el que se encuentra en la dieta diaria de los panameños. Para los aspectos pertinentes se utilizan los datos estadísticos generados por el Instituto Nacional de Estadística y Censo (INEC) de la Contraloría General de La República de Panamá. Se desarrolla un análisis de los datos generados por medio de estadísticas descriptivas, correlación de variales y regresión.

Actualmente el sector agrícola de Panamá está confrontando una situación difícil en la producción de arroz; para el caso de la Provincia de Chiriquí las estimaciones de la producción se determinan empíricamente en base a promedios de años anteriores, lo que dificulta determinar los requerimientos del producto para cubrir la demanda nacional y el momento adecuado para precisar las importaciones de ser necesario y para no perjudicar a los productores de este sector.

Lo anterior permite formular la siguiente interrogante: ¿Qué relación existe entre la producción de arroz en Chiriquí y la superficie sembrada como un aporte al consumo nacional? 


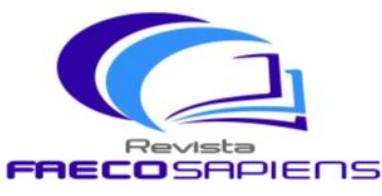

Revista ค月EடロS月คIERS
ताT Volumen 3 - Número 1 Enero-Junio 2020

Recibido: 18/10/19 Aceptado: 25/11/19 pp. 21-40 Indexada

\section{METODOLOGÍA}

En este apartado se muestran los aspectos más importantes para lograr los objetivos propuestos de forma que se pueda validar la hipótesis alterna planteada.

Se presenta una investigación de tipo correlacional que tiene como finalidad conocer el grado de asociación que existe en la producción de arroz y la superficie sembrada. "La utilidad principal de los estudios correlacionales es saber cómo se puede comportar un concepto o una variable al conocer el comportamiento de otras variables vinculadas" (Hernández Sampieri, Fernández Collado, \& Baptista Lucio, 2010, pág. 82).

El estudio es de tipo correlacional no experimental, busca evidencias de la relación que existe en la variable producción de arroz en función de la superficie sembrada. Tiene un componente longitudinal porque utiliza datos estadísticos de los años 1,990 $-2017$.

La fuente de información la conforma los datos publicados por el INEC de la Contraloría General de la República de Panamá. Se escogieron los datos sobre la producción de arroz y la superficie sembrada en la Provincia de Chiriquí del año 1990 al 2017 por ser estos de data reciente, lo que pueden representar un comportamiento más real para predecir.

La "hipótesis es una suposición o solución anticipada al problema objeto de la investigación y, por tanto, la tarea del investigador debe orientarse a probar tal suposición" (Bernal Torres, 2010, pág. 136).

La hipótesis que se pretende evaluar es:

Ho: $\mathbf{p}=\mathbf{0}$ (No existe correlación lineal en la cantidad de arroz cosechada $\rightarrow$ variable 


$$
\mathrm{Y} \leftarrow \text {, y la superficie sembrada } \rightarrow \text { variable } \mathrm{X} \leftarrow) \text {. }
$$

H1: $\mathbf{p} \neq \mathbf{0}$ (Existe correlación lineal en la cantidad de arroz cosechada $\rightarrow$ variable

$$
\mathrm{Y} \leftarrow \text {, y la superficie sembrada } \rightarrow \text { variable } \mathrm{X} \leftarrow \text { ) }
$$

La prueba de hipótesis se realizará mediante el análisis de tipo paramétrico con el estadístico de prueba de correlación de Pearson (r) y el estadístico de DurbinWatson, ya que el propósito de esta investigación es analizar la relación entre dos variables, con un nivel de significancia de $95 \%(P=0.05)$.

Las variables representan las características que poseen los individuos o sujetos de una población estudiada, pueden ser cualitativa - descriptiva o cuantitativa que puede medirse.

Para el caso se estudian las variables superficie sembrada $(X)$ y la variable producción de arroz generada $(\mathrm{Y})$.

Se utiliza una recta de regresión lineal que se ajusta a la nube de puntos estudiados, donde la variable $Y$ está en función de $X ; Y=f(X)$, descrito con la ecuación $Y=\beta_{1}+\beta_{2} X$, donde $\beta_{1}$ es la ordenada al origen y $\beta_{2}$ corresponde a la pendiente. Los cálculos se han estimado con el software Excel y Statistical Package for the Social Sciences, (SPSS). 


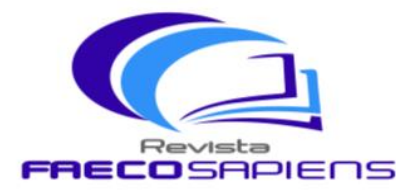

Revista मECロ
ताT Volumen 3 - Número 1

Enero-Junio 2020

Recibido: 18/10/19 Aceptado: 25/11/19 pp. 21-40 Indexada

\section{RESULTADOS}

En este apartado se presentan los resultados logrados utilizando las técnicas estadísticas siguientes.

La Estadística Descriptiva tiene como función el manejo de los datos recopilados en cuanto se refiere a su ordenación y presentación, para poner en evidencia ciertas características en la forma que sea más objetiva y útil (Morales, 2012). Se trata de encontrar las medidas que sinteticen las distribuciones de frecuencias.

Las medidas de tendencia central más representativas son: media aritmética, mediana y moda (Posada Hernández, 2016). Para sintetizar el manejo de los datos, podemos caracterizar su distribución de frecuencias mediante algunos valores numéricos. Esto se logra, eligiendo en el sumario de los datos un valor central, alrededor del cual se encuentren distribuidos los valores de la variable.

El valor de la variable elegida para representar una distribución se llama media o promedio, y es un valor representativo de todos los valores que toma la variable. La media debe hallarse entre el menor y el mayor valor de la variable. En consecuencia, se han estimado las medidas de tendencia central de la estructura de los datos o de la muestra utilizada en la investigación. Además, se procedió a calcular las medidas de dispersión y las de forma.

La Media: Se calcula sencillamente sumando los distintos valores de la variable estudiada y dividiendo por el número de datos (Gorgas García, Cardiel López, \& Zamorano Calvo, 2011). La media de la cosecha de arroz para el periodo de estudio es de 2,270,140.036 y la media de la superficie sembrada es de 27,347.3214 hectáreas. (ver Tabla 1).

La Mediana: Se define como una medida central tal que, con los datos ordenados de menor a mayor, el $50 \%$ de los datos son inferiores a su valor y el $50 \%$ de los datos tienen valores superiores (Gorgas García, Cardiel López, \& Zamorano Calvo, 2011). El valor que divide la serie en partes iguales, es decir la mediana para 


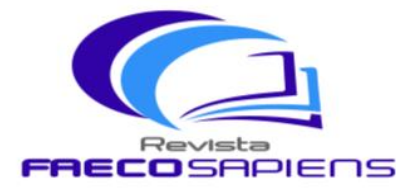

Revista

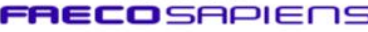

Volumen 3 - Número 1
Enero-Junio 2020

Recibido: 18/10/19 Aceptado: 25/11/19 pp. 21-40 Indexada

la superficie sembrada es 23,460 hectáreas y la mediana para la cosecha de arroz es 1,927,100 quintales (Tabla 1).

La Moda: La moda de una muestra es aquel valor de la variable que tiene una frecuencia máxima. En otras palabras, es el valor que más se repite. Hay que indicar que puede suceder que la moda no sea única, es decir que aparezcan varios máximos en la distribución de frecuencias, (Gorgas García, Cardiel López, \& Zamorano Calvo, 2011). Para el caso de la superficie sembrada no existe moda ya que no se repite ningún valor y para la cosecha de arroz sucede los mismo no se repite valores (\#N/A). (Tabla 1).

La dispersión estudia la distribución de los valores de la serie, analizando si estos se encuentran más o menos concentrados, o más o menos dispersos, (USAC, 2011). permiten calcular la representatividad de una medida de posición, para lo cual será preciso cuantificar la distancia de los diferentes valores de la distribución respecto a dicha medida. A tal distancia es lo que, en términos estadísticos, denominaremos variabilidad o dispersión de la distribución. Las medidas de dispersión tienen como finalidad estudiar hasta qué punto, para una determinada distribución de frecuencias, las medidas de tendencia central son representativas como síntesis de toda la información de la distribución.

Rango: Es la diferencia entre la observación más alta y la más baja de una muestra de datos, también, la diferencia entre el valor máximo y el valor mínimo, es decir el rango de la superficie sembrada $\mathbf{3 0 , 4 2 0}$ y el rango de la coseche de arroz es 2,958,200 (Tabla 1).

Varianza: Es el promedio de las desviaciones respecto a su media elevadas al cuadrado. Nunca puede ser negativa. La Varianza asume el valor de $69,825,402.9$ para la superficie sembrada y $611,526,180,711.74$ para la cosecha de 


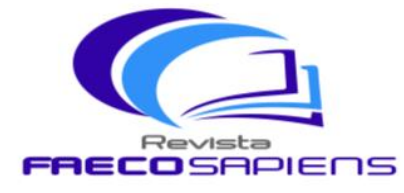

Revista

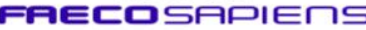

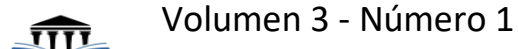

Enero-Junio 2020

Recibido: 18/10/19 Aceptado: 25/11/19 pp. 21-40 Indexada

arroz; lo cual indica que la superficie sembrada ha variado en $69,825,402.9$ y la cosecha de arroz en $611,526,180,711.74$. (Tabla 1).

Desviación Estándar: La desviación estándar indica la distribución de los datos alrededor de la media aritmética o promedio, (Posada Hernández, 2016). La desviación estándar es considerada la medida de dispersión con mayor representatividad para un conjunto de datos. Es la raíz cuadrada de la varianza. La Desviación Estándar determinada para la superficie sembrada es 8,356.2 y para la

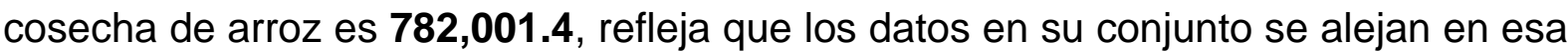
proporción de la media. (Tabla 1).

Las medidas de forma son usadas para identificar valores particulares de un conjunto de datos. Para ello toman como referencia la media aritmética y la desviación estándar de la población o la muestra, (Posada Hernández, 2016). Las medidas de forma de una distribución basan su representación en una gráfica, sin llegar a realizar la misma. Las medidas de forma se clasifican en medidas de asimetría y medidas de curtosis o apuntamiento.

Curtosis: Las medidas de curtosis estudian la distribución de frecuencias en la zona central de la misma. La mayor o menor concentración de frecuencias alrededor de la media y en la zona central de la distribución dará lugar a una distribución más o menos apuntada. La Curtosis calculada para la superficie

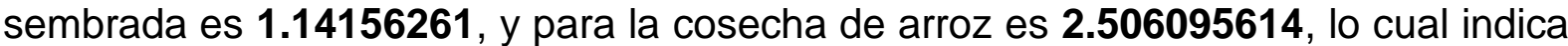
que las distribuciones muestrales tienen una forma de campana con una cresta pronunciada por encima del eje de las $\mathrm{X}$; tienen una distribución de tipo leptocúrtica. (Tabla 1).

Coeficiente de asimetría: Las medidas de asimetría tienen como finalidad elaborar un indicador que permita establecer el grado de simetría o asimetría que presenta una distribución de frecuencias. El Coeficiente de Asimetría para la superficie sembrada es 1.44823659 , y para la cosecha de arroz es 1.71174015 , valor que 


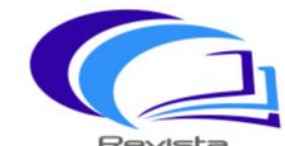

Revista

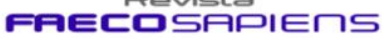

शTV Volumen 3 - Número 1 DRJI Enero-Junio 2020

Recibido: 18/10/19 Aceptado: 25/11/19 pp. 21-40 Indexada

indica que la distribución es asimétrica positiva, es decir, la distribución está inclinada hacia la derecha. (Tabla 1 y Figuras 1, 2 ).

\begin{tabular}{|c|c|c|c|}
\hline \multicolumn{4}{|c|}{$\begin{array}{c}\text { Tabla } 1 \\
\text { Medidas de Tendencia central, dispersión y forma }\end{array}$} \\
\hline \multicolumn{2}{|c|}{$\begin{array}{c}\text { Superficie sembrada de arroz } \\
\text { (hectáreas) }\end{array}$} & \multicolumn{2}{|c|}{$\begin{array}{c}\text { Cosecha de arroz en cáscara } \\
\text { (quintales) }\end{array}$} \\
\hline Media & 27347.3214 & Media & 2270140.036 \\
\hline Error típico & 1579.16573 & Error típico & 147784.3724 \\
\hline Mediana & 23460 & Mediana & 1927100 \\
\hline Moda & \#N/D & Moda & $\# N / D$ \\
\hline Desviación estándar & 8356.15958 & Desviación estándar & 782001.3943 \\
\hline Varianza de la muestra & 69825402.9 & Varianza de la muestra & 611526180711.74 \\
\hline Curtosis & 1.14156261 & Curtosis & 2.506095614 \\
\hline Coeficiente de asimetría & 1.44823659 & Coeficiente de asimetría & 1.71174015 \\
\hline Rango & 30420 & Rango & 2958200 \\
\hline Mínimo & 18080 & Mínimo & 1585000 \\
\hline Máximo & 48500 & Máximo & 4543200 \\
\hline Suma & 765725 & Suma & 63563921 \\
\hline Cuenta & 28 & Cuenta & 28 \\
\hline
\end{tabular}

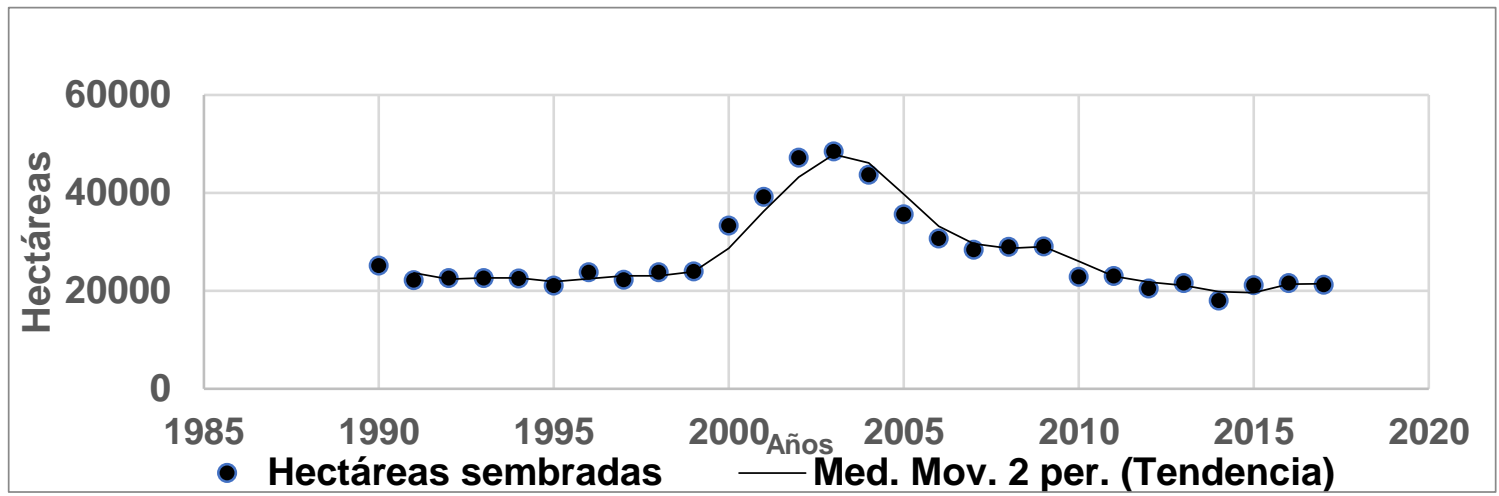

Figura 1. Hectáreas sembradas de arroz en la Provincia de Chiriquí 1990 -2017. Fuente: Cálculos Propios 


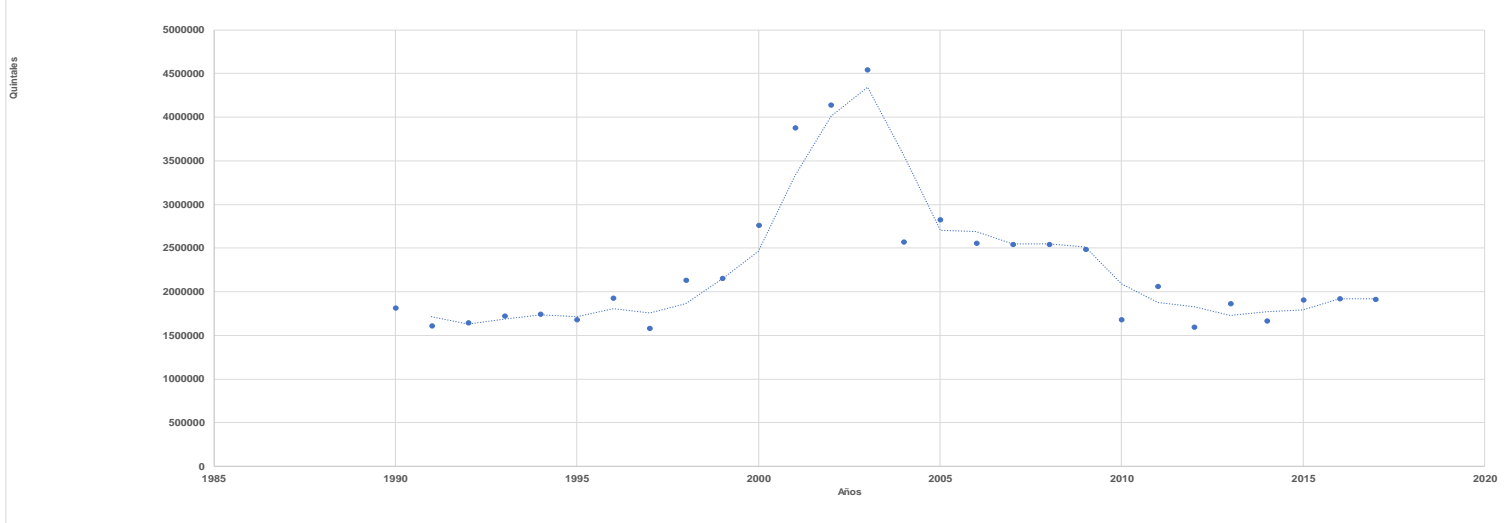

Figura 2. Cosecha de arroz en la Provincia de Chiriquí 1990-2017. Fuente: Cálculos Propios

"Un gráfico de Pareto es un ordenado histograma con una línea adicional que describe cómo cada grupo contribuye al total” (Cronquist, 2018).

Para el análisis correspondiente se presentan los datos de la superficie sembrada y la cosecha de arroz que corresponden a los años 1990 - 2017, con un total de 28 datos observados. El valor máximo para las hectáreas sembradas es de 48,500 y para la cosecha de arroz es 4,543,200 quintales, mientras que el valor mínimo identificado para la superficie sembrada es de 18,080 y para la cosecha de arroz es $\mathbf{1 , 5 8 5 , 0 0 0}$. En la variable superficie sembrada, el intervalo 24,164 concentra la mayor frecuencia, que indica que los productores chiricanos por lo regular siembran 24,164 hectáreas o menos de arroz por año. Además, se han construido los gráficos de Pareto para mostrar el comportamiento de las frecuencias. Para la variable dependiente, cosecha de arroz, el intervalo 2,176,640 concentra la mayor frecuencia, que nos muestra que los productores están cosechando por lo regular 2,176,640 quintales de arroz por año, (Tabla 2 y 3) y (Figuras 3 y 4) 
REVISTA FAECO SAPIENS

ISSN L 2644-3821

Acceso Abierto. Disponible en:

https://revistas.up.ac.pa/index.php/faeco sapiens

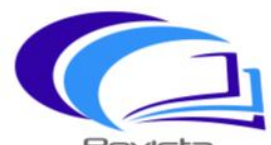

Reviste

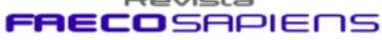

गाT Volumen 3 - Número 1 DRJI
Recibido: 18/10/19 Aceptado: 25/11/19 pp. 21-40 Indexada

Tabla 2

\begin{tabular}{|c|c|c|c|c|}
\hline \multicolumn{5}{|c|}{ Datos para el diagrama de Pareto - Hectáreas sembradas - } \\
\hline INTERVALOS & FRECUENCIA & $\%$ ACUM & PORCENTAJES & FREC ACUM \\
\hline 24164 & 17 & $60.71 \%$ & $60.71 \%$ & 17 \\
\hline 30248 & 4 & $75.00 \%$ & $14.29 \%$ & 21 \\
\hline 36332 & 3 & $85.71 \%$ & $10.71 \%$ & 24 \\
\hline 48500 & 3 & $89.29 \%$ & $3.57 \%$ & 27 \\
\hline 42416 & 1 & $100.00 \%$ & $10.71 \%$ & 28 \\
\hline TOTAL & 28 & & $100.00 \%$ & \\
\hline Fuente: Cálculos propios & \multicolumn{4}{|l}{} \\
\hline
\end{tabular}

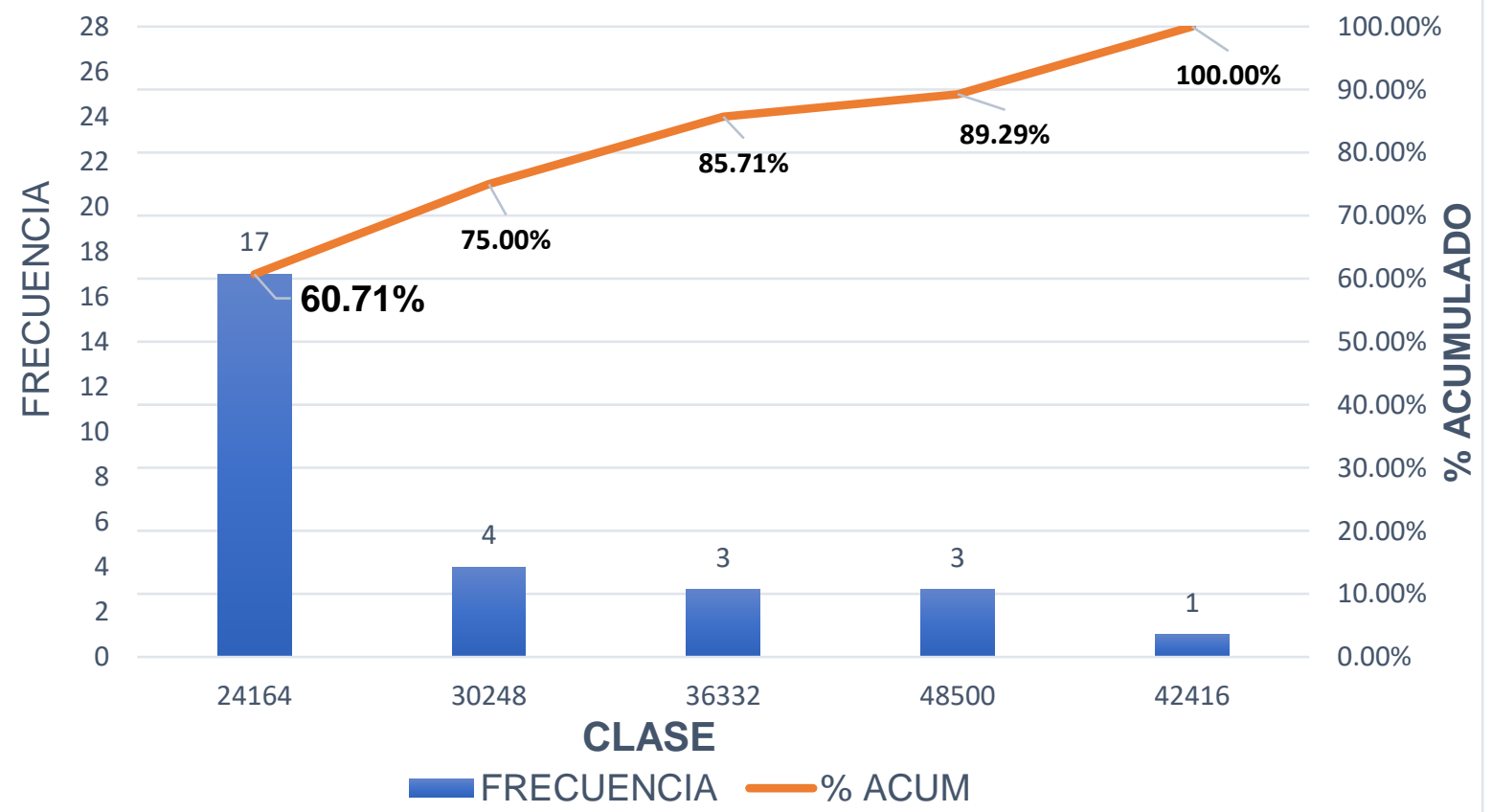

Figura 3. Diagrama Pateto: Variación de las hectáreas sembradas 1990-2017. Fuente: Cálculos propios 
REVISTA FAECO SAPIENS

ISSN L 2644-3821

Acceso Abierto. Disponible en:

https://revistas.up.ac.pa/index.php/faeco sapiens

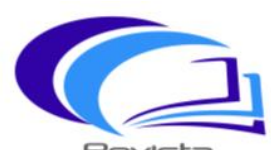

Revista

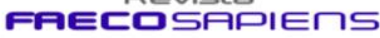

गTा Volumen 3 - Número 1 DRJI

Recibido: 18/10/19 Aceptado: 25/11/19 pp. 21-40 Indexada

\section{Tabla 3}

Datos para el diagrama de Pareto - Cosecha de arroz -

\begin{tabular}{|c|c|c|c|c|}
\hline INTERVALOS & FRECUENCIA & $\%$ ACUM & PORCENTAJES & FREC ACUM \\
\hline 2176640 & 18 & $64.29 \%$ & $64.29 \%$ & 18 \\
\hline 2768280 & 6 & $85.71 \%$ & $21.43 \%$ & 24 \\
\hline 4543200 & 2 & $92.86 \%$ & $7.14 \%$ & 26 \\
\hline 3359920 & 1 & $96.43 \%$ & $3.57 \%$ & 27 \\
\hline 3951560 & 1 & $100.00 \%$ & $3.57 \%$ & 28 \\
\hline TOTAL & 28 & & $100.00 \%$ & \\
\hline
\end{tabular}

Fuente: Cálculos propios

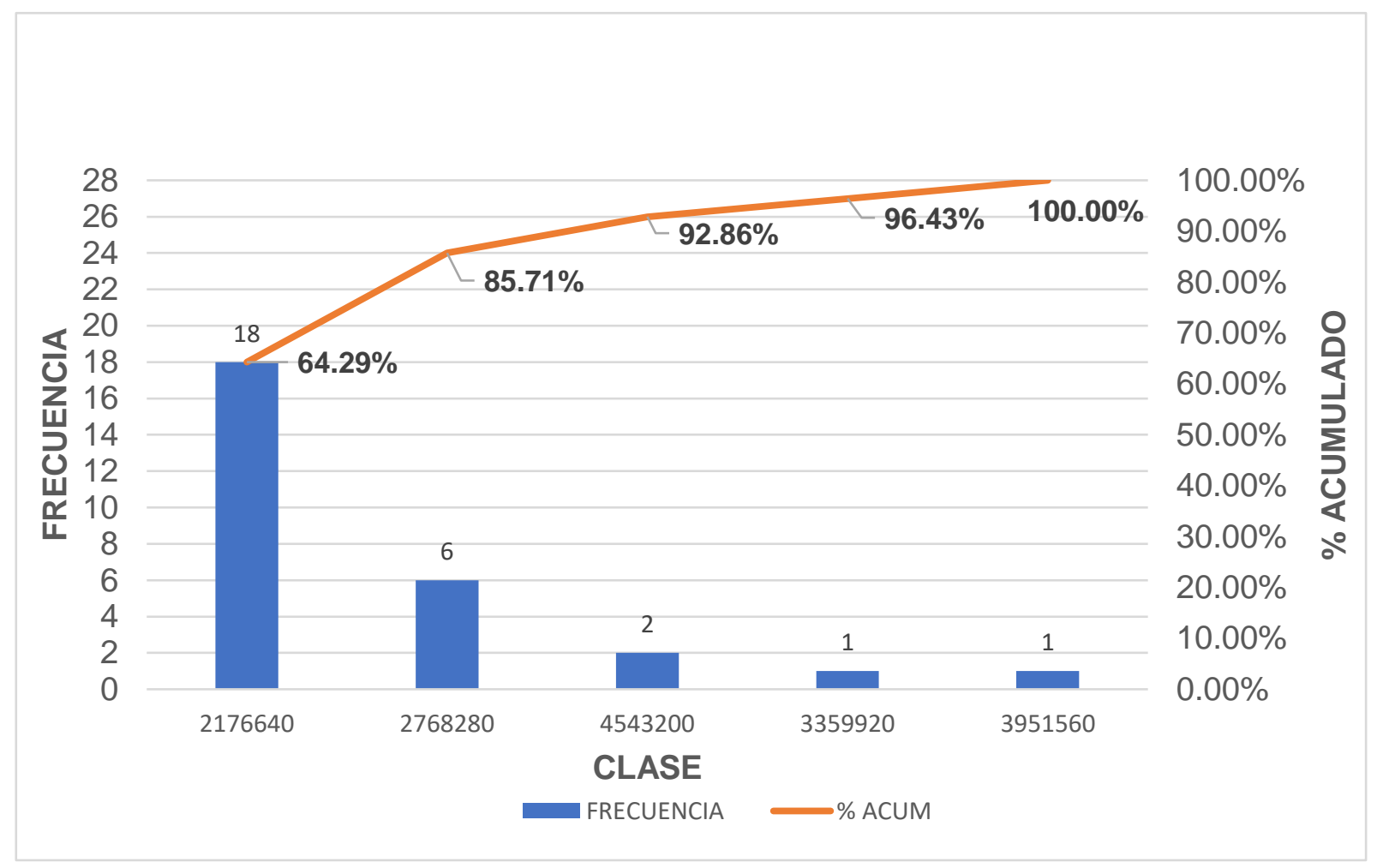

Figura 4. Diagrama de Pareto: Variación de la cosecha de arroz 1990-2017. Fuente: Cálculos propios 


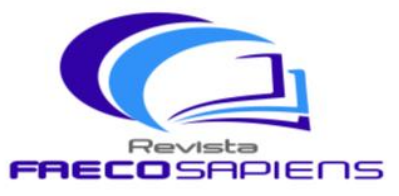

गाT Volumen 3 - Número 1 DRJI Enero-Junio 2020

Para los efectos de relacionar el comportamiento de la superficie sembrada y la cosecha de arroz, se han extraído los datos del INEC, Contraloría General de la República de Panamá, a través de la URL de internet https://www.contraloria.gob.pa/inec/Publicaciones/Publicaciones.aspx?ID SUBCATE GORIA=11\&ID PUBLICACION=852\&ID IDIOMA=1\&ID CATEGORIA=4

En el Figura 5 podemos observar el comportamiento y la relación de la variable superficie sembrada vs cosecha de arroz.

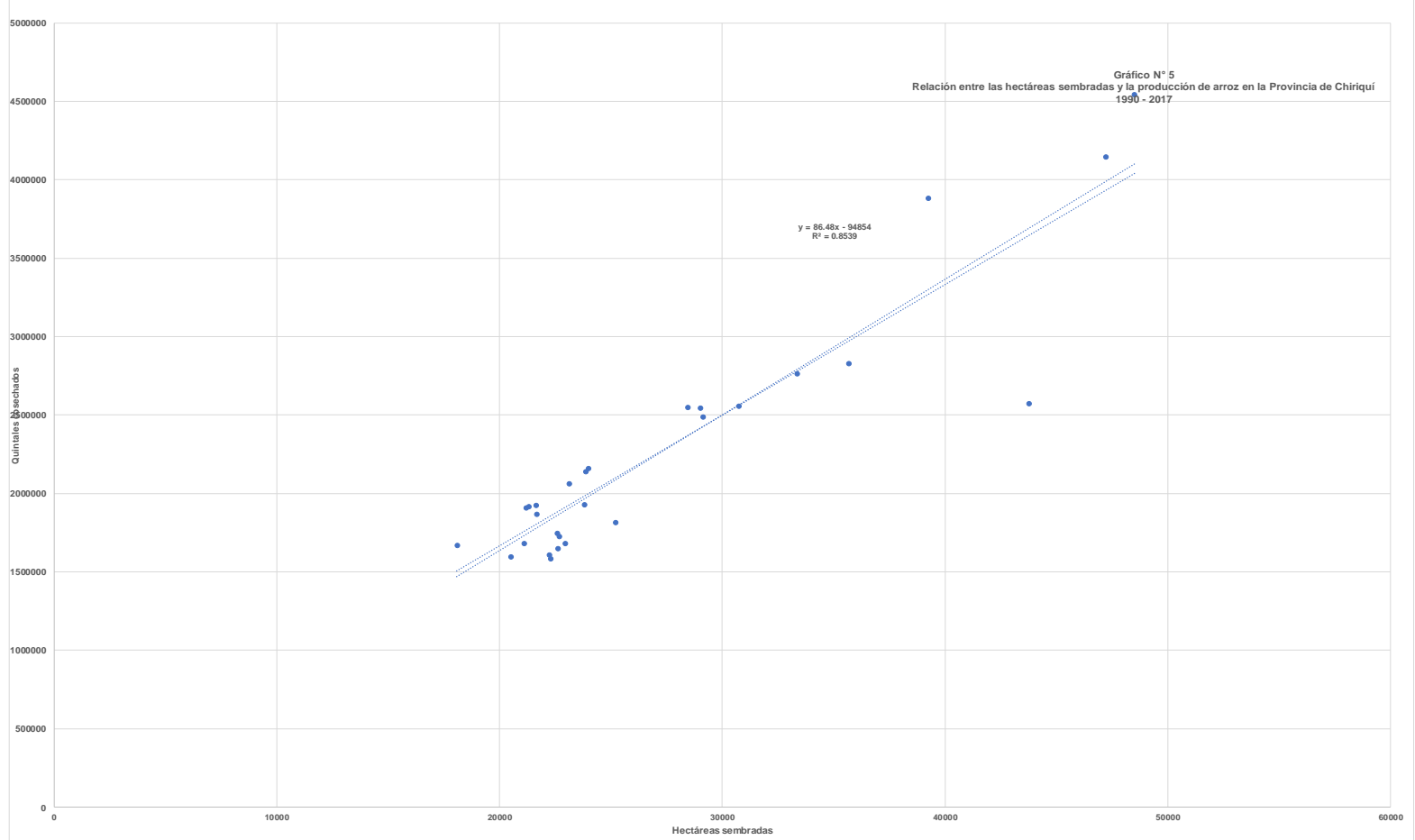

Figura 5. Relación entre la producción de las hectáreas sembradas y la producción de arroz. Fuente: Cálculos Propios

Para determinar la relación que existe entre la variable dependiente e independiente se planteó la hipótesis $\left(\mathrm{H}_{1}\right)$ que el valor de los quintales de arroz cosechado está 


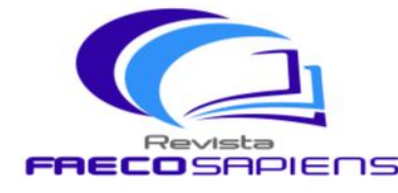

Revista ค月EடロS月คIEחS
ताT Volumen 3 - Número 1

Enero-Junio 2020

Recibido: 18/10/19 Aceptado: 25/11/19 pp. 21-40 Indexada

correlacionado con el valor de la superficie sembrada dentro del sistema agrícola de la Provincia de Chiriquí.

El Figura 5 describe el comportamiento de la cosecha de arroz en cáscara medido en quintales en función de la superficie sembrada (hectáreas). Es decir, el $\mathrm{R}^{2}$ mide la proporción o porcentaje de la variación total en $\mathrm{Y}$ explicada por el modelo de regresión, (Gujarati, 1981). La función matemática muestra un elevado grado de bondad en el ajuste de la función, sustentado por el valor de $\mathbf{R}^{\mathbf{2}} \mathbf{\mathbf { 0 . 8 5 3 9 }}$, es decir la variable superficie sembrada explica en un $\mathbf{8 5 . 3 9 \%}$ el valor de la cosecha de arroz. Por otra parte, la línea de tendencia, que se obtiene a partir de la función lineal, $\mathrm{Y}($ Cosecha de arroz $)=\mathbf{8 6 . 4 8 X}$ (Superficie sembrada $)$ - 94854, nos describe una ecuación con pendiente positiva que se ajusta al comportamiento de la distribución de los datos y a los valores del coeficiente de asimetría y curtosis calculados.

El coeficiente de correlación lineal simple mide el grado de asociación lineal entre dos variables, (Alegre Martín \& Cladera Munar, 2002). Como se ha planteado, para los efectos de comparar el comportamiento de la superficie sembrada en la Provincia de Chiriquí, con una segunda variable la cosecha de arroz se ha procedido a escoger los datos del INEC, presentados en el Anexo. Con las herramientas del software Excel y SPSS se ha calculado la Correlación, a fin de describir la relación entre ambas variables.

El coeficiente de correlación (r), se calcula para determinar si dos conjuntos de datos varían conjuntamente de forma directamente proporcional (correlación positiva), inversamente proporcional (correlación negativa), o si no están correlacionados (correlación con un valor cercano a cero, sin tendencia observable). El valor $\mathbf{r}$ $=\mathbf{0 . 9 2 4}$, señala que los datos estudiados tienen un alto grado de correlación entre las variables de cosecha de arroz y la superficie sembrada, con una correlación positiva o directamente proporcional. (Tabla 5). 


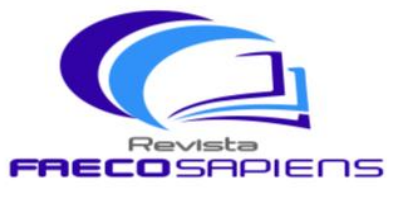

\begin{tabular}{|c|c|c|c|}
\hline \multicolumn{4}{|c|}{$\begin{array}{c}\text { Tabla } 5 \\
\text { Coeficiente de correlación }\end{array}$} \\
\hline & & Cosecha de $\operatorname{arroz}(\mathrm{Y})$ & Hectáreas sembradas $(X)$ \\
\hline Correlación de & Cosecha de arroz $(\mathrm{Y})$ & 1.000 & .924 \\
\hline Pearson & Hectáreas sembradas $(X)$ & .924 & 1.000 \\
\hline
\end{tabular}

La Hipótesis de investigación es: La cantidad de arroz cosechada en la Provincia de Chiriquí depende de la superficie sembrada.

El resumen de las estadísticas de la Regresión lineal, indican un alto grado de correlación entre la variable dependiente (Cosecha de arroz) y la variable independiente (superficie sembrada), sobre la base del Coeficiente de Correlación $(r)=0.924 ;$ y la bondad en el ajuste de la recta de regresión que es igualmente alto, dado por el coeficiente de determinación, $\mathbf{R}^{\mathbf{2}}=\mathbf{0 . 8 5 4}$ mientras que el error típico es de 308,606.36. (Tabla 6)

\begin{tabular}{|l|r|}
\hline \multicolumn{2}{|c|}{ Tabla 6} \\
\hline Coeficiente de correlaciósticas de la regresión $(r)$ & 0.924 \\
\hline Coeficiente de determinación $\mathrm{R}^{\wedge} 2$ & 0.854 \\
\hline $\mathrm{R}^{\wedge} 2$ ajustado & 0.848 \\
\hline Error estándar de la estimación & 28 \\
\hline Observaciones & $204,553.9$ \\
\hline Fuente: Cálculos propios \\
\hline
\end{tabular}

La hipótesis que se formuló y que se contrasta a continuación es:

Ho: $\mathbf{p}=\mathbf{0}$ (No existe correlación lineal en la cantidad de arroz cosechada $\rightarrow$ variable $\mathrm{Y} \leftarrow$, y la superficie sembrada $\rightarrow$ variable $\mathrm{X} \leftarrow$ ).

H1: $\mathbf{p} \neq \mathbf{0}$ (Existe correlación lineal en la cantidad de arroz cosechada $\rightarrow$ variable 


$$
\mathrm{Y} \leftarrow, \text { y la superficie sembrada } \rightarrow \text { variable } \mathrm{X} \leftarrow \text { ) }
$$

Para validar la hipótesis planteada se utilizó la tabla de valores críticos del coeficiente de correlación de Pearson $-r-$, con un alfa $=0.05$ y $n=28$.

La Figura $\mathbf{N}^{\circ} 1$ muestra las zonas de rechazo y no rechazo de la hipótesis nula; como el valor del coeficiente de correlación $r=0.924$ cae en la zona de rechazar la hipótesis nula (Ho: no existe correlación entre las variables cosecha de arroz y hectáreas sembradas) se acepta la hipótesis alterna ( $\mathrm{H}_{1}$ : existe correlación entre las variables cosecha de arroz y hectáreas sembradas), concluye que las variables están altamente correlacionadas de forma directamente proporcional.

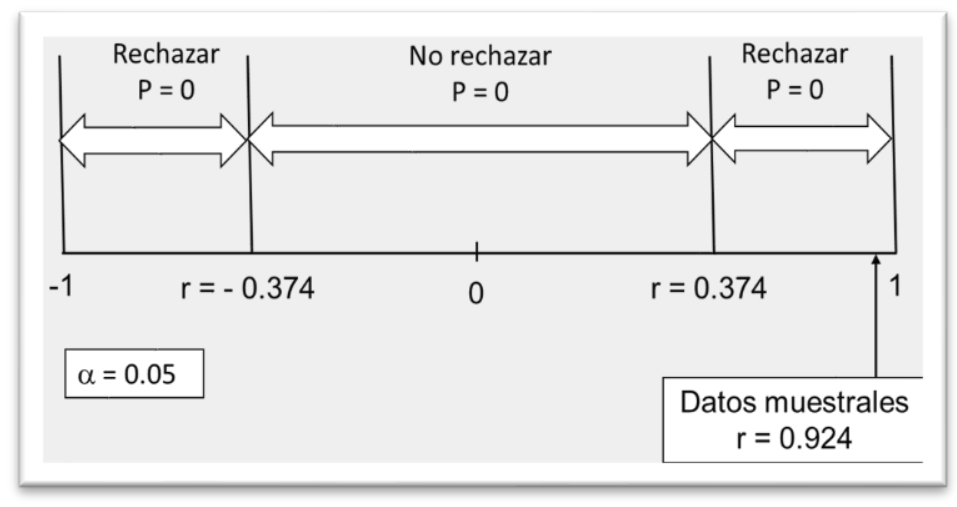

Figura 6. Modelo de aceptación y rechazo de hipótesis según coeficiente $r$ de Pearson

Para la evaluación del modelo se utilizó el programa de la compañía IBM Corporation, SPSS 25.0 que es un software profesional utilizado para hacer cálculos matemáticos y estadísticos. Para el modelo se utilizó el P-Valor o Significancia y el test de Durbin Watson.

El P-Valor es una prueba de significancia cuyo valor tiene que ser contrastado con el error de investigación que es de $5 \%$. El valor de significancia tiene que ser menor que el error (0.05). Dado que el P-valor de significancia obtenido es de 0.000 , la hipótesis planteada se acepta. (Tabla 7). 


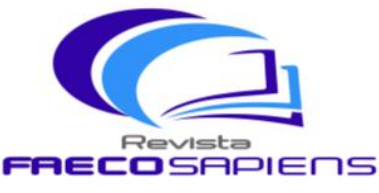

गाT Volumen 3 - Número 1 DRJI Enero-Junio 2020

Recibido: 18/10/19 Aceptado: 25/11/19 pp. 21-40 Indexada

Las variables pueden estar altamente correlacionadas, pero pueden tener problemas de autocorrelación. El test de Durbin Watson (D.W.) permite evaluar si existe auto correlación en una regresión lineal, sea simple o múltiple, con ello se pretende determinar si el modelo de regresión $(Y=86.48 X-94854)$ es lo suficientemente robusto para hacer predicciones. El valor del coeficiente D.W. puede estar dentro de un rango de $\mathbf{0}$ a 4 . El límite de aceptación para el modelo de regresión está dentro de los parámetros 1.476 a 2.524. Como el valor $\mathbf{d}=$ 1.918 está dentro del área de aceptación se puede confirmar que el modelo es robusto para hacer predicciones. (Tabla 7 y Figura 8 ).

\begin{tabular}{|c|c|c|c|c|c|c|c|c|c|c|}
\hline \multicolumn{11}{|c|}{ Tabla 7} \\
\hline \multicolumn{11}{|c|}{ Resumen del modelo ${ }^{b}$} \\
\hline \multirow[b]{2}{*}{ Modelo } & \multirow[b]{2}{*}{$\mathrm{R}$} & \multirow[b]{2}{*}{$\begin{array}{c}\mathrm{R} \\
\text { cuadrado } \\
\end{array}$} & \multirow[b]{2}{*}{\begin{tabular}{|c|}
$\mathrm{R}$ \\
cuadrado \\
ajustado \\
\end{tabular}} & \multirow[b]{2}{*}{$\begin{array}{c}\text { Error } \\
\text { estándar de } \\
\text { la estimación }\end{array}$} & \multicolumn{5}{|c|}{ Estadísticos de cambio } & \multirow[b]{2}{*}{$\begin{array}{l}\text { Durbin- } \\
\text { Watson }\end{array}$} \\
\hline & & & & & $\begin{array}{c}\text { Cambio } \\
\text { en } \mathrm{R} \\
\text { cuadrado }\end{array}$ & $\begin{array}{c}\text { Cambio } \\
\text { en } F\end{array}$ & gl1 & $\mathrm{gl} 2$ & \begin{tabular}{|c} 
Sig. \\
Cambio \\
en $\mathrm{F}$ \\
\end{tabular} & \\
\hline 1 & $.924^{\mathrm{a}}$ & .854 & .848 & 304553.9 & .854 & 152.012 & 1 & 26 & .000 & 1.918 \\
\hline \multicolumn{11}{|c|}{ a. Predictores: (Constante), Hectáreas sembradas } \\
\hline \multicolumn{11}{|c|}{ b. Variable dependiente: Cosecha de arroz } \\
\hline Fuente: & 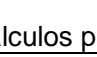 & oios & & & & & & & & \\
\hline
\end{tabular}

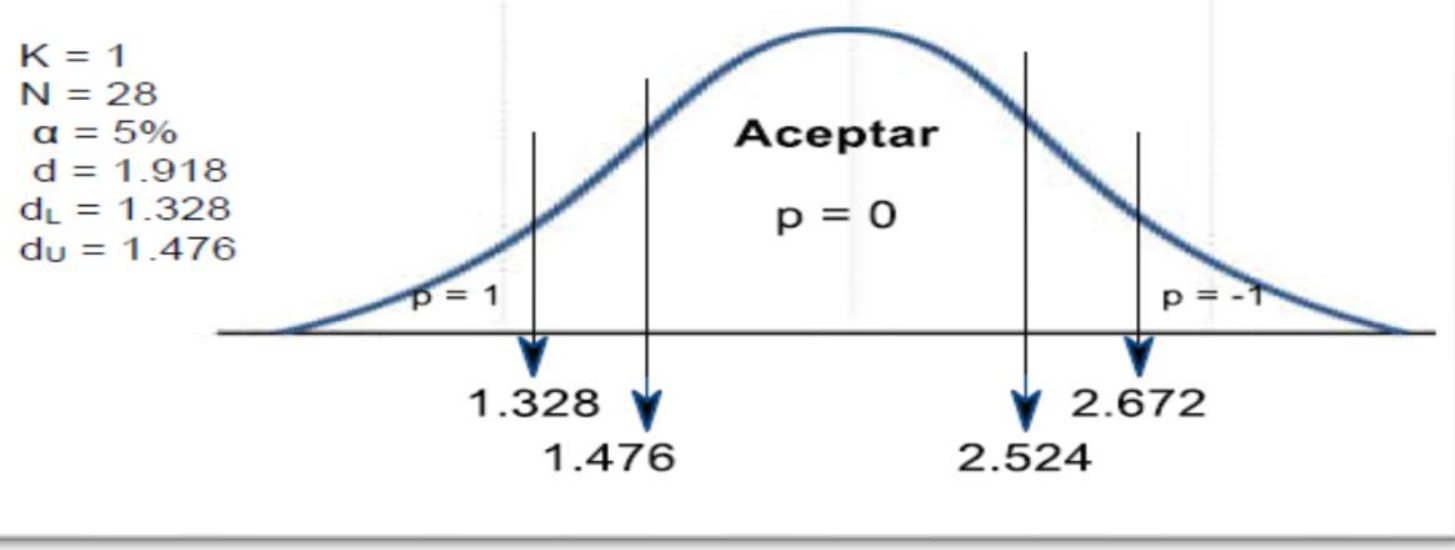

Figura 8. Modelo de aceptación y rechazo de hipótesis según Durbin Watson. Fuente: Cálculos propios 


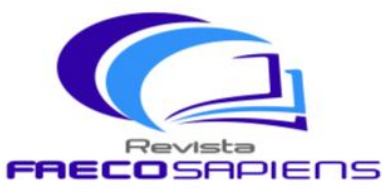

TाT Volumen 3 - Número 1

Para el año 2017 la producción de arroz en Panamá no llenó las expectativas.

“...el consumo de arroz diario de los panameños es de 20,000 quintales pilados, lo que equivale a 27,397 en cascara, de acuerdo con la Federación de Arroceros y Granos de Panamá." (Panamá América, 2017). Con esta afirmación se concluye que el consumo anual de este alimento en Panamá es de 9,999,905 quintales en cáscara aproximadamente, de los cuales la Provincia de Chiriquí aporta 1,917,300 en el periodo $2017 / 2018$ quintales que representa $19.17 \%$.

\section{CONCLUSIONES}

Se efectuó una revisión de la base de datos de las estadísticas del INEC de la Contraloría General de la República de Panamá, para buscar la relación que existe entre la superficie sembrada y la cosecha de arroz en la Provincia de Chiriquí, con el propósito de diseñar un modelo que permita a los productores predecir la producción anual de arroz en cascara. Esta información es de relevancia para el Gobierno Nacional; le permite planificar un programa de asistencia económica para fortalecer el sector y programar las importaciones (de ser necesario) sin perjudicar al productor.

La investigación permite hacer las siguientes afirmaciones sobre la producción de arroz en Chiriquí.

- En promedio se siembran 27,347 hectáreas por año (según periodo de estudio).

- En la variable superficie sembrada, el intervalo 24,164 concentra la mayor frecuencia, que indica que los productores chiricanos por lo regular siembran 24,164 hectáreas o menos de arroz.

- En promedio se cosecha 2,270,140 quintales de arroz en cáscara por año (según periodo de estudio).

- Se acepta la hipótesis de investigación que la producción de arroz (variable Y) está altamente correlacionada con la superficie sembrada (variable $\mathrm{X}$ ) con un valor $r=0.924$. 


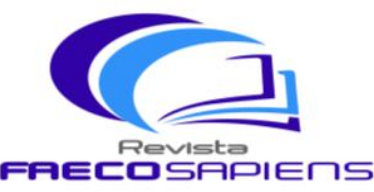

- La función de producción $\mathrm{Y}=86.48 X-94854$ es robusta para predecir la producción de arroz, según la superficie sembrada, ya que carece de problemas de autocorrelación, según coeficiente D.W. = 1.918.

- La producción de arroz en Chiriquí hace un aporte significativo al consumo Nacional con un $19.17 \%$.

\section{REFERENCIAS BIBLIOGRÁFICAS}

Alegre Martín, J., \& Cladera Munar, M. (2002). INTRODUCCIÓN A LA ESTADÍSTICA DESCRIPTIVA PARA ECONOMISTAS. Palma de Malloeca: Universitat de les Illes Balears.

Angulo, S. (30 de Septiembre de 2017). expreso.ec. Obtenido de http://www.expreso.ec/economia/importaciones-comercio-economia-bancocentral-CA1736271

Bernal Torres, C. A. (2010). Metodología de la investigación. Bogotá: Pearson.

Cronquist, O. (29 de septiembre de 2018). Cómo crear un gráfico de Pareto. Obtenido de https://www.get-digital-help.com/es/c\%C3\%B3mo-crear-un-gr\%C3\%A1fico-de-Pareto/

El Panamá América. (23 de octubre de 2017). Panamá cubre su demanda de arroz con importaciones. La producción nacional de Arroz se encuentra amenazada ante las constantes importaciones, muchas veces sin necesidad.

Gómez Sánchez, A. M., \& Ramírez Gutiérrez, Z. (2016). CAUSALIDAD ENTRE LAS IMPORTACIONES Y EL CRECIMIENTO ECONÓMICO: EVIDENCIA EMPÍRICA PARA EL DEPARTAMENTO DEL CAUCA (COLOMBIA). UAEM redal.yc.org.

GÓMEZ SÁNCHEZ, A. M., \& RAMÍREZ GUTIÉRREZ, Z. (Diciembre, 2017). CAUSALIDAD ENTRE LAS IMPORTACIONES Y EL CRECIMIENTO ECONÓMICO: EVIDENCIA EMPÍRICA PARA EL DEPARTAMENTO DEL CAUCA (COLOMBIA). Revista Facultad de Ciencias Económicas: Investigación y Reflexión, 47-48-49-50-51-52-53-54-55.

Gorgas García, J., Cardiel López, N., \& Zamorano Calvo, J. (2011). Estadística Básica para Estudiantes de Ciencias. Madrid, España: Universidad Complutense.

Gujarati, D. (1981). Econometría Básica. New York: Mc Graw Hill.

INGENIERÍA, U. F. (2011). Manual de Estadística Descriptiva. Guatemala.

Lasso, Mileika;. (23 de Abril de 2017). Panamá declara al arroz, cultivo de seguridad alimentaria. La Estrella de Panamá. Obtenido de http://laestrella.com.pa/economia/panama-declara-arrozcultivo-seguridad-alimentaria/23997595 


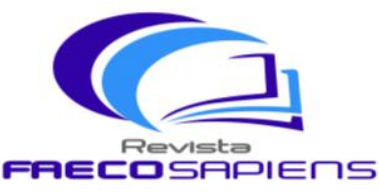

गTा Volumen 3 - Número 1 DRJI Enero-Junio 2020

Recibido: 18/10/19 Aceptado: 25/11/19 pp. 21-40 Indexada

Montesino, J. L. (2007). LA ECONOMÍA ECUATORIANA DEL SIGLO XXI Y SUS PERSPECTIVAS DE COMERCIO INTERNACIONAL CON PAIISES DEL ASIA PACÍFICO. Si Somos Americanos, Revista de Estudios Transfronterizos, 79-80-81-82-83-84-85-86-87.

Morales, G. Aaron Estuardo;. (2012). Estadística y Probabilidades. Concepción, Chile: Universidad Católica de la Santísima Concepción.

Morales, R. (08 de Junio de 2017). El Economista. Obtenido de https://www.eleconomista.com.mx/empresas/Mexico-pais-que-mas-PIB-gasta-para-comprarproductos-de-EU-20170609-0006.html

Panamá América. (23 de octubre de 2017). Panamá cubre su demanda de arroz con importaciones.

Pendino, S. (s.f.). sebastiánpendino. Obtenido de Ley de Pareto: Dispara tu Productividad con la Regla 80/20: https://sebastianpendino.com/ley-de-pareto-productividad/

Posada Hernández, G. (2016). ELEMENTOS BÁSICOS DE ESTADÍSTICA DESCRIPTIVA PARA EL ANÁLISIS DE DATOS. Medellín, Colombia: Fondo Editorial Luís Amigo.

Sánchez Navas, M. A. (s.f.).

Sánchez Navas, M. A. (s.f.). Monografías.com. Obtenido de http://www.monografias.com/trabajos108/estudio-relacion-del-pib-importaciones-panama1946-2015/estudio-relacion-del-pib-importaciones-panama-1946-2015.shtml

USAC. (2011). Manual de EStadística Descriptiva. Guatemala.

Xiaoying Li, D. G. (Julio-Agosto de 2005). MPORTACIONES DE SERVICIOS Y CRECIMIENTO ECONÓMICO. Obtenido de http://www.revistasice.com/CachePDF/ICE_824_723_2DB8E1FEDA08E6F8248036256ECEA7D5.pdf

Xiaoying Li, D. G. (Julio-Agosto 2005). MPORTACIONES DE SERVICIOS Y CRECIMIENTO ECONÓMICO. UN ANÁLISIS DINÁMICO DE PANEL. ICE, 15-16-17. 


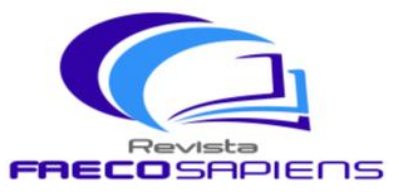

गाT Volumen 3 - Número 1 DRJI Enero-Junio 2020

Recibido: 18/10/19 Aceptado: 25/11/19 pp. 21-40 Indexada

ANEXO

Tabla de Datos Provincia de Chiriqui SUPERFICIE (Hectáreas) y COSECHA DE ARROZ (Quintales)

\begin{tabular}{|l|l|l|l|l|l|}
\hline \multicolumn{5}{|c|}{$\mathbf{1 9 9 0} \mathbf{2 0 1 6}$} \\
\hline Años & SUPERFICIE & COSECHA & Años & SUPERFICIE & COSECHA \\
\hline 1990 & 25,203 & $1,815,097$ & 2004 & 43,750 & $2,574,200$ \\
\hline 1991 & 22,210 & $1,611,000$ & 2005 & 35,660 & $2,830,300$ \\
\hline 1992 & 22,620 & $1,651,400$ & 2006 & 30,740 & $2,556,500$ \\
\hline 1993 & 22,660 & $1,726,400$ & 2007 & 28,440 & $2,547,900$ \\
\hline 1994 & 22,590 & $1,748,900$ & 2008 & 29,000 & $2,544,300$ \\
\hline 1995 & 21,100 & $1,681,100$ & 2009 & 29,120 & $2,489,900$ \\
\hline 1996 & 23,810 & $1,928,700$ & 2010 & 22,939 & $1,683,722$ \\
\hline 1997 & 22,280 & $1,585,000$ & 2011 & 23,110 & $2,065,000$ \\
\hline 1998 & 23,870 & $2,138,300$ & 2012 & 20,500 & $1,595,600$ \\
\hline 1999 & 23,970 & $2,160,200$ & 2013 & 21,660 & $1,869,400$ \\
\hline 2000 & 33,343 & $2,766,402$ & 2014 & 18,080 & $1,670,000$ \\
\hline 2001 & 39,250 & $3,883,900$ & 2015 & 21,170 & $1,909,500$ \\
\hline 2002 & 47,220 & $4,145,200$ & 2016 & 21,630 & $1,925,500$ \\
\hline 2003 & 48,500 & $4,543,200$ & 2017 & 21,300 & $1,917,300$ \\
\hline Fuente: Contrán General de la República de Panamá - INEC & \\
\hline
\end{tabular}

1 - La producción de arroz corresponde desde agosto a enero del siguiente año. "Se consideró la producción como si corresponde al año previo".

2 - Del año 2010/2011 en adelante incluye las comarcas Kuna Yala, Emberá y Ngäbe Buglé.

El primer periodo de cosecha de arroz en Panamá es de agosto a octubre y el segundo, de noviembre a enero, según datos del Ministerio de Desarrollo Agropecuario. 